\title{
STRONG SURJECTIONS AND NEARNESS
}

\author{
ADRIANA BUICA
}

Dedicated to Alfonso Vignoli on the ocasion of his 60th birthday

Summary: We show that the properties of being strongly surjective or stable solvable (in the sense of Furi-Martelli-Vignoli) carry over to maps which are near in the sense of Campanato.

Keywords: Near mappings, strong surjection, stable solvable map, implicit differential equations.

Classification: 47H99, 47H15, 34A09, 34B15.

1. Introduction. M. Furi, M. Martelli and A. Vignoli in [3] introduced the notions of strong surjection and stable solvable map between two normed spaces $E$ and $F$ in order to define the spectrum for a nonlinear operator. Also, these concepts are related to that of zero-epi map, which is due to the same authors [4] and is very important in the study of solvability of nonlinear equations.

Near operators have been introduced by S. Campanato and also studied by A. Tarsia and S. Leonardi in $[2,7,10,13,14]$ and have applications in nonlinear differential equations, too.

We prove that the property of being a strong surjection or stable solvable is preserved by nearness and notice that this can be used in order to prove existence results for differential equations in implicit form.

2. Main results. Let $E$ be a normed space and $F$ be a Banach space. A continuous map $f: E \rightarrow F$ is called a strong surjection if the equation $f(x)=h(x)$ has a solution for any continuous compact map $h: E \rightarrow F$.

A continuous map $f: E \rightarrow F$ is said to be stable solvable if the equation $f(x)=h(x)$ has a solution for any completely continuous map $h: E \rightarrow F$ 
with quasinorm $|h|=0$. Recall that the quasinorm of a map $h$ is defined by

$$
|h|=\limsup _{\|x\| \rightarrow \infty} \frac{\|h(x)\|}{\|x\|} .
$$

We say that $g: E \rightarrow F$ is near $f: E \rightarrow F$ if there exist two positive constants $\alpha$ and $k$, with $k \in(0,1)$, such that we have

$$
\left\|f\left(x_{1}\right)-f\left(x_{2}\right)-\alpha\left[g\left(x_{1}\right)-g\left(x_{2}\right)\right]\right\| \leq k\left\|f\left(x_{1}\right)-f\left(x_{2}\right)\right\|
$$

for all $x_{1}, x_{2} \in E$.

In order to prove our main results we shall give two lemmas.

Lemma 1 Let $g: E \rightarrow F$ be near $f: E \rightarrow F$. If $f$ is continuous, then $g$ is continuous, too.

Proof. Using that $g$ is near $f$ we obtain the following estimation.

$$
\begin{aligned}
& \left\|g\left(x_{1}\right)-g\left(x_{2}\right)\right\|= \\
= & \frac{1}{\alpha}\left\|f\left(x_{1}\right)-f\left(x_{2}\right)-\alpha\left[g\left(x_{1}\right)-g\left(x_{2}\right)\right]-\left[f\left(x_{1}\right)-f\left(x_{2}\right)\right]\right\| \leq \\
\leq & \left(\frac{k}{\alpha}+1\right)\left\|f\left(x_{1}\right)-f\left(x_{2}\right)\right\|
\end{aligned}
$$

for all $x_{1}, x_{2} \in E$.

Now, by the definition of the continuity we deduce that, if $f$ is continuous, then $g$ is also continuous.

In what follows we shall denote by $f_{d}^{-1}$ a right inverse for a surjective map $f$.

Lemma 2 Let $g: E \rightarrow F$ be near a surjective map $f: E \rightarrow F$. The following statements are true.

(i) $f(x)=f(\hat{x})$ implies that $g(x)=g(\hat{x})$

(ii) $s=(f-\alpha g) \circ f_{d}^{-1}: F \rightarrow F$ is a contraction and does not depend on the choice of the right inverse of $f$.

Proof. If we consider $x, \hat{x} \in E$ with $f(x)=f(\hat{x})$ and replace in (1) we obtain $\|g(x)-g(\hat{x})\| \leq 0$, which means that $g(x)=g(\hat{x})$. From this we can deduce that $s$ does not depend on the choice of $f_{d}^{-1}$.

The following estimation is obtained using (1) and express that $s$ is a contraction.

$$
\begin{aligned}
& \left\|(f-\alpha g)\left(f_{d}^{-1} y_{1}\right)-(f-\alpha g)\left(f_{d}^{-1} y_{2}\right)\right\| \leq \\
\leq & k\left\|f\left(f_{d}^{-1} y_{1}\right)-f\left(f_{d}^{-1} y_{2}\right)\right\|=k\left\|y_{1}-y_{2}\right\|
\end{aligned}
$$


for all $y_{1}, y_{2} \in F$.

Remark. Relation (1) express also that the map $f-\alpha g$ is a contraction with respect to $f$. For other considerations in this direction we recommend $[1,6,13]$.

Theorem 1 Let $g: E \rightarrow F$ be near $f: E \rightarrow F$. If $f$ is a strong surjection, then $g$ is a strong surjection, too.

Proof. By Lemma 1, $g$ is a continuous map. Let $h: E \rightarrow F$ be continuous and compact. By Lemma 2 we have that $s=(f-\alpha g) \circ f_{d}^{-1}: F \rightarrow F$ is a contraction, where $f_{d}^{-1}$ is a right inverse for $f$. In this situation we have that $(I-s)$ is a homeomorphism.

The map $(I-s)^{-1} \circ \alpha h: E \rightarrow F$ is continuous and compact. So, by hypothesis it has a coincidence point with the strong surjection $f$, i.e. $f(x)=(I-s)^{-1}(\alpha h(x))$. Let us denote $\hat{x}=f_{d}^{-1}(f(x))$ and notice that $f(x)=f(\hat{x})$ and $g(x)=g(\hat{x})$. The following implications are valid.

$$
\begin{aligned}
& f(x)=(I-s)^{-1}(\alpha h(x)) \Longrightarrow(I-s)(f(x))=\alpha h(x) \Longrightarrow \\
& f(x)-(f-\alpha g)(\hat{x})=\alpha h(x) \Longrightarrow g(x)=h(x)
\end{aligned}
$$

This means that $x$ is a coincidence point of $g$ and $h$, where $h$ is an arbitrary continuous and compact map. So, $g$ is a strong surjection.

Theorem 2 Let $g: E \rightarrow F$ be near $f: E \rightarrow F$. If $f$ is stable solvable, then $g$ is stable solvable, too.

Proof. Let $h: E \rightarrow F$ be completely continuous with quasinorm $|h|=0$. The arguments follow like in the previous theorem, noticing that $(I-s)^{-1}$ 。 $\alpha h$ is completely continuous with quasinorm equal to 0 .

3. An application. Let us consider two mappings $L, N: E \rightarrow F$ such that $L-N$ is a strong surjection. In applications (see $[4,5,8,11]$ ), usually, $L$ is linear and bounded (in many cases, a differential operator) and $N$ is completely continuous. In the case that $\|(L-N)(x)\| \rightarrow \infty$ as $\|x\| \rightarrow$ $\infty$ there are some relations between the theory of strong surjections and the theory of zero-epi maps, or degree theory, or the theory of essential compact fields (see $[3,4,7,9]$ ). Using this, we can find many examples of strong surjections of the form $L-N$. One of them which is due to M. Furi, 
M. Martelli and A. Vignoli [4] is the following.

Let $C_{0}^{2}[0,1]$ be the space of $C^{2}$-functions such that $x(0)=x(1)=0$ and $L, N: C_{0}^{2}[0,1] \rightarrow C[0,1]$ be defined by $L x(t)=x^{\prime \prime}(t)$ and $N(x)(t)=x^{3}(t)$. Then $L-N$ is a strong surjection.

We use our main results in order to state that a map of the (implicit) form

$$
g: E \rightarrow F, \quad g(x)=G(L x, N(x))
$$

is a strong surjection provided that $L-N$ is a strong surjection and $G$ : $F \times F \rightarrow F$ satisfies the following relation for some $\alpha>0$ and $k \in(0,1)$ and for all $y_{1}, y_{2}, z_{1}, z_{2} \in F$

$$
\left\|y_{1}-z_{1}-y_{2}+z_{2}-\alpha\left[G\left(y_{1}, z_{1}\right)-G\left(y_{2}, z_{2}\right)\right]\right\| \leq k\left\|y_{1}-z_{1}-y_{2}+z_{2}\right\| .
$$

For example, $g: C_{0}^{2}[0,1] \rightarrow C[0,1]$ defined by $g(x)(t)=\tilde{g}\left(x^{\prime \prime}(t), x^{3}(t)\right)$ is a strong surjection if $\tilde{g}: \mathbb{R} \times \mathbb{R} \rightarrow \mathbb{R}$ satisfies (2) for all $y_{1}, y_{2}, z_{1}, z_{2} \in \mathbb{R}$.

\section{References}

[1] A. BUICA: Data dependence theorems on coincidence problems, Studia UBB 41(1996), 33-40

[2] S. CAMPANATO: Further contribution to the theory of near mappings, Le Matematiche 48(1993), 183-187

[3] M. FURI, M. MARTELLI, A. VIGNOLI: Contributions to the spectral theory for nonlinear operators in Banach spaces, Annali Mat. Pura Appl. 118(1978), 229-294

[4] M. FURI, M. MARTELLI, A. VIGNOLI: On the solvability of nonlinear operator equations in normed spaces, Annali Mat. Pura Appl. 124(1980), 321-343

[5] R. E. GAINES, J. MAWHIN: The Coincidence Degree and Nonlinear Differential Equations, Lect. Notes Math. 568, Springer, Berlin 1977

[6] K. GOEBEL: A coincidence theorem, Bull. Acad. Pol. Sci. 16(1968), 733-735

[7] A. GRANAS: The theory of compact vector fields and some of its applications to topology of functional spaces I, Rozprawy Mat. 30, Warsaw (1962) 
[8] A. GRANAS, R. B. GUENTHER, J. W. LEE: Some general principles in the Caratheodory theory of nonlinear differential systems, J. Math. Pures Appl. 70(1991), 153-196

[9] D. H. HYERS, G. ISAC, T. M. RASSIAS: Topics in Nonlinear Analysis and Applications, World Scientific, Singapore 1997

[10] S. LEONARDI: On the Campanato nearness condition, Le Matematiche 48(1993), 179-181

[11] R. PRECUP: Existence theorems for nonlinear problems by continuation methods, Nonlin. Anal. TMA 30(1997), 3313-3322

[12] I. A. RUS: Some remarks on coincidence theory, Pure Math. Manuscr. $\mathbf{9}(1990 / 91), 137-148$

[13] A. TARSIA: Some topological properties preserved by nearness between operators and applications to PDE's, Czechosl. Math. J. 46(121)(1996), 607-624

[14] A. TARSIA: Differential equations and implicit functions: a generalization of the near operators theorem, Topol. Methods Nonlin. Anal. 11(1998), 115-133

ADRIANA BUICA, Department of Applied Mathematics, Babes-Bolyai University, Str. Kogalniceanu 1, RO-3400 Cluj-Napoca, Romania; abuica@math.ubbcluj.ro 